\title{
Evaluation of human embryo development in in vitro fertilization- and intracytoplasmic sperm injection- fertilized oocytes: A time-lapse study
}

\author{
Hyung Jun Kim', Hye Jin Yoon', Jung Mi Jang', Won Don Lee', San Hyun Yoon², Jin Ho Lim² \\ 'Maria Fertility Hospital, Seoul; ${ }^{2}$ Fertility Research Center, Maria Medical Foundation, Seoul, Korea
}

Objective: We investigated whether the insemination method (in vitro fertilization [IVF] or intracytoplasmic sperm injection [ICSI]) affected morphokinetic events and abnormal cleavage events in embryonic development.

Methods: A total of 1,830 normal fertilized embryos were obtained from 272 IVF and ICSI cycles that underwent ovum retrieval culture using a time-lapse system (Embryoscope) from June 2013 to March 2015. All embryos were investigated by a detailed time-lapse analysis that measured the developmental events in the hours after IVF or ICSI insemination.

Results: No significant differences were observed between the two groups regarding clinical outcomes $(p>0.05)$. ICSI-derived embryos showed significantly faster morphokinetics than those derived from conventional IVF, from the time to pronuclear fading to the time to 6 cells $(p<0.05)$. However, no significant differences were found from the time to 7 cells to the time to expanded blastocyst $(p>0.05)$. There were no differences in abnormal cleavage events between the two groups ( $p>0.05)$; they showed the same rates of direct cleavage from 1 to 3 cells, 2 multinucleated cells, 2 uneven cells, and reverse cleavage.

Conclusion: The morphokinetics of embryo development was found to vary between IVF- and ICSI-fertilized oocytes, at least until the 6-cell stage. However, these differences did not affect the clinical outcomes of the embryo. Additionally, no significant differences in abnormal cleavage events were found according to the fertilization method.

Keywords: In vitro fertilization; Insemination; Intracytoplasmic sperm injection; Time-lapse imaging

\section{Introduction}

Since the first in vitro fertilization-embryo transfer (IVF-ET) baby was born in 1978, IVF-ET procedures have made remarkable progress, and various assisted reproductive technologies have been implemented. Unfortunately, the routine embryo selection method used during the IVF-ET procedure remains unsatisfactory; thus, most IVF centers induce a higher pregnancy rate by implanting more than one embryo.

Received: Mar 17, 2017· Revised: Apr 8, 2017· Accepted: May 17, 2017

Corresponding author: Hyung Jun Kim

Maria Fertility Hospital, 20 Cheonho-daero, Dongdaemun-gu, Seoul 02586, Korea

Tel: +82-2-2250-5522 Fax:+82-2-2250-5549 E-mail: blue@mariababy.com

This is an Open Access article distributed under the terms of the Creative Commons Attribution Non-Commercial License (http://creativecommons.org/licenses/by-nc/4.0/) which permits unrestricted non-commercial use, distribution, and reproduction in any medium, provided the original work is properly cited.
Multiple ET increases the chance of conception from IVF, but it is associated with an increased chance of multiple pregnancies [1]. Multiple pregnancies have been reported to result in obstetrical and neonatal complications, such as congenital malformations, low birth weight with intrauterine growth retardation, and cesarean sections [2,3]. The elective transfer of a single embryo has been suggested as the most efficient approach to avoid multiple pregnancies [4]. Therefore, embryo selection is a very important process before ET. Embryo selection based on a morphological assessment at a few points in time has several limitations for single ET. Images of the embryo provide more accurate data to guide embryo selection $[5,6]$.

Time-lapse monitoring systems enable the detailed evaluation of morphology, including dynamic parameters, and they allow the exclusion of negative factors, such as multi-nucleation of the blastomere and irregular division. Several methods have been proposed 
for culturing embryos using a time-lapse incubator to maintain a stable environment. Observation of embryos in a time-lapse incubator can provide useful information about embryonic developmental events if images are automatically captured [7-10].

In 1997, Payne et al. [11] developed the first time-lapse system for studying morphokinetics in human embryos. This system overcame the limitations of intermittent observation. Their research group has described the timing of the morphological events of fertilization, including extrusion of the polar body and formation of the pronucleus, using their video recording system.

Approximately 10 years later, Mio [12] developed a new system for time-lapse monitoring, that maintains optimal stable culture conditions for long periods, based on the report of Payne et al. [11]. This new technique can take more than 2,000 images during the early stages of human embryonic development and provides useful information about embryonic development after intracytoplasmic sperm injection (ICSI) for approximately 40 hours, when embryos reach the 2 to 4 cell stage. Formation of the morula and blastocyst hatching were successfully monitored in vitro for 2 to 5 days [13].

In a more recent study, a model was developed to predict embryo implantation based on the timing and characteristics of cleavage events, further underscoring the usefulness of continued embryo observation [10].

The intrinsic difference between ICSI and IVF is known to affect the zygote and to affect embryo development in general [14,15]. IVF requires a sperm cell to penetrate the cumulus cells and the zona pellucida, but ICSI can induce insemination without certain processes taking place. Moreover, ICSI is more invasive than conventional IVF. For example, ICSI oocytes are exposed to hyaluronidase and intense light during the denuding process and are damaged by mechanical pipetting [16].

It was found that ICSI-fertilized 4-cell embryos spent approximately 2.5 hours less time in the 2-cell stage than IVF-fertilized 4-cell embryos, and that the 3-cell stage was longer in ICSI-fertilized oocytes [7]. In addition, the first cleavage has been reported to be slow in conventional IVF [17]. However, the small sample size used in those two studies limits the analysis of the morphokinetics of embryo cleavage, making it difficult to compare insemination techniques.

This study compared insemination methods (IVF and ICSI) to determine whether they had different effects on morphokinetics and abnormal cleavage events in embryonic development.

\section{Methods}

\section{Patients and design}

This study included 272 cycles of IVF/ICSI treatment from June 2013 to March 2015 at Maria Fertility Hospital. The study was approved by the Institutional Review Board of Maria Fertility Hospital (No. 2016005). The patients included had no chromosomal diseases and had normal karyotypes.

\section{Ovarian stimulation and oocyte retrieval}

Women were treated with a gonadotropin-releasing hormone agonist (Superfact; Sanofi-Aventis, Frankfurt, Germany) using the long protocol or a gonadotropin-releasing hormone antagonist protocol and recombinant follicle-stimulating hormone (Gonal F; Merck Serono, Darmstadt, Germany). When two or more follicles reached 18 $\mathrm{mm}$ in diameter, 10,000 IU of human chorionic gonadotropin (hCG) (IVF-C; LG Chemical, Seoul, Korea) or Ovidrel (Merck Serono) was administered.

\section{Conventional IVF}

IVF insemination was performed by adding $1 \times 10^{6}$ spermatozoa/ $\mathrm{mL}$. Fertilization was assessed 16 to 18 hours after insemination and confirmed based on the presence of two pronuclei and two polar bodies. Fertilized embryos were cultured using a pre-equilibrated EmbryoSlide (Unisense FertiliTech, Aarhus, Denmark).

\section{Intracytoplasmic sperm injection}

ICSI was usually performed when the sperm sample was poor, based on the embryologist's judgment and following the World Health Organization criteria of sperm concentration $<15 \times 10^{6} / \mathrm{mL}$, motility $<40 \%$, and strict morphology $<4 \%$.

After removing the cumulus cell attached to the egg with MRC\#ICSI (Biosupply, Seoul, Korea) containing $0.1 \%$ hyaluronidase (Biosupply), the collected oocytes were cultured in Sydney IVF fertilization medium (Cook, Brisbane, Australia). ICSI was performed using an inverted Nikon microscope (TE 2000 U; Nikon, Kawasaki, Japan) equipped with a manipulator (Narishige, Tokyo, Japan). Sperm selection was performed under $\times 200$ to $\times 400$ magnification, and microinjection of each oocyte was performed as described by Kim et al. [18]. Once injected, the oocytes were placed in individual wells of a pre-equilibrated EmbryoSlide (Unisense FertiliTech).

\section{Culture and annotation using time-lapse monitoring}

For culture with the EmbryoScope (Vitrolife, Goteborg, Sweden), dedicated 12-well plates were prepared with $25-\mu \mathrm{L}$ microdrops of Sydney IVF cleavage medium (Cook) overlaid by $1.4 \mathrm{~mL}$ of mineral oil (Biosupply) at $37^{\circ} \mathrm{C}$ in $6 \% \mathrm{CO}_{2}, 5 \% \mathrm{O}_{2}$, and $89 \% \mathrm{~N}_{2}$. The surplus embryos developed to the blastocyst stage were vitrified by the electron microscopy-grid method following artificial shrinkage of the blastocoel [19].

Images of each embryo were analyzed retrospectively using the EmbryoViewer external image analysis software (Unisense Fertili- 
Tech). Images were acquired every 20 minutes in seven different focal planes during culture [10]. Embryonic developmental events were annotated with the corresponding timing in units of hours after IVF and ICSI. Annotations included the time to faded pronuclei (tPNf), the times to a 9-cell embryo (t2, t3, t4, t5, t6, t7, t8, and t9), time to formation of a morula (tM), initiation of blastulation (tSB), the formation of the blastocyst (tB), and time taken to complete the maximal blastocyst expansion (tEB). Cell cycle durations were calculated for the second cell cycle $(c c 2, t 3-t 2)$, the third cell cycle $(c c 3, t 5-t 3)$, the synchrony of the second cell cycle (s2, t4-t3), and the synchrony of the third cell cycle (s3, t8-t5). Additionally, abnormal cleavage events, multinucleation, and the good-quality embryo rate per embryo on day 3 were investigated. Good-quality embryos were defined as having 8 to 10 cells, no embryo fragmentation, and cells that were very even, regular, and similarly sized.

\section{Confirmation of pregnancy}

If $\beta$-hCG was detected in the blood 14 days after egg collection, the trace test after 1 week showed a continuous increase, and vaginal ultrasonography performed at 6 to 7 weeks of pregnancy showed a gestational sac, then clinical pregnancy was verified. The implantation rate was calculated as the percentage of embryonic sacs implanted in the uterus that became implanted embryos. Miscarriage was defined as a fetal loss prior to 12 weeks of gestation despite the presence of a gestational sac in the first-trimester ultrasound.

\section{Statistical analysis}

Statistical analysis was performed with SPSS ver. 12.0 (SPSS Inc., Chicago, IL, USA). Quantitative data are presented as the mean \pm standard deviation, and properties are shown for categorical variables. The Student $t$-test was used to compare continuous variables,

Table 1. Characteristics of the patients

\begin{tabular}{lccc}
\hline Characteristics & IVF & ICSI & $p$-value \\
\hline No. of cycles & 122 & 150 & - \\
Female age $(\mathrm{yr})$ & $33.2 \pm 2.3$ & $33.5 \pm 2.4$ & 0.524 \\
BMI $\left(\mathrm{kg} / \mathrm{m}^{2}\right)$ & $21.3 \pm 2.6$ & $21.4 \pm 3.0$ & 0.603 \\
Basal FSH (IU/L) & $6.7 \pm 2.0$ & $6.9 \pm 2.2$ & 0.502 \\
AMH (ng/mL) & $4.7 \pm 2.8$ & $4.9 \pm 3.1$ & 0.346 \\
Previous ART cycles & $0.6 \pm 0.9$ & $0.5 \pm 0.7$ & 0.353 \\
Thickness of endometrium (mm) & $8.7 \pm 1.0$ & $8.9 \pm 1.1$ & 0.342 \\
Triggering & & & \\
$\quad$ GnRH agonist & 82 & 93 & 0.372 \\
GnRH antagonist & 40 & 57 & 0.372 \\
\hline
\end{tabular}

Values are mean \pm standard deviation.

IVF, in vitro fertilization; ICSI, intracytoplasmic sperm injection; BMI, body mass index; FSH, follicle-stimulating hormone; $\mathrm{AMH}$, anti-Müllerian hormone; ART, assisted reproductive technology; $G n R H$, gonadotropin-releasing hormone. and the chi-square test was used for discrete variables. The $p$-values $<0.05$ were considered to indicate statistical significance.

\section{Results}

This study included 272 cycles of IVF/ICSI treatments (122 conventional IVF and 150 ICSI). When the characteristics of the IVF cycles were compared with those of the ICSI cycles, no significant differences were found in female age, body mass index, basal follicle-stimulating hormone, previous assisted reproductive technology cycles, or endometrial thickness (Table 1). Comparisons of the clinical outcomes between the two groups are presented in Table 2. No significant differences were observed between the two groups with regard to the retrieved oocytes, fertilization rate, the high-quality embryo rate on day 3 , and the number of transferred embryos. Furthermore, no significant differences were found between IVF and ICSI regarding the clinical pregnancy rate ( $57.4 \%$ vs. $52.0 \%)$, implantation rate (33.2\% vs. $30.5 \%)$, or miscarriage rate (15.1\% vs. $16.3 \%$ ) (Table 2$)$.

ICSI-derived embryos developed significantly faster than those produced with conventional IVF at tPNf $(25.2 \pm 4.2$ hours vs. $24.3 \pm 3.9$ hours, $p<0.001)$, t2 ( $28.1 \pm 4.8$ hours vs. $27.0 \pm 4.5$ hours, $p<0.001)$, t3 $(37.1 \pm 6.1$ hours vs. $36.5 \pm 5.7$ hours, $p=0.021)$, $\mathrm{t} 4$ (39.4 \pm 6.2 hours vs. $38.7 \pm 5.8$ hours, $p=0.016), \mathrm{t} 5$ ( $49.9 \pm 8.8$ hours vs. $46.7 \pm 7.9$ hours, $p=0.005)$, and t6 (53.4 \pm 9.6 hours vs. $52.4 \pm 8.5$ hours, $p=0.030$ ). However, no significant differences were found from t7 to tEB between the two groups. The cell cycle of cc2 was not different, but the IVF group ( $12.9 \pm 6.3$ hours vs. $11.9 \pm 6.9$ hours, $p=0.001)$ had a longer cycle than ICSI for cc3. No statistically significant differences were observed in the synchrony of the cell cycles (s1, s2, and s3) (Table 3).

We compared abnormal cleavage events in embryonic development. There were no differences between the two groups in the rates of direct cleavage from 1 to 3 cell $(p=0.149), 2$ multinucleated cells $(p=0.691), 2$ uneven cells ( $p=0.463)$, or reverse cleavage $(p=0.884)$ (Table 4).

Table 2. Comparison of laboratory and clinical outcome measures according to the fertilization method

\begin{tabular}{lccc}
\hline Variable & IVF & ICSI & $p$-value \\
\hline No. of cycles & 122 & 150 & - \\
No. of retrieved oocytes & $9.4 \pm 3.4$ & $9.6 \pm 4.1$ & 0.767 \\
Fertilization rate & $69.8 \pm 16.9$ & $72.8 \pm 17.5$ & 0.093 \\
Good-quality embryo rate on day 3 & $55.2 \pm 29.9$ & $58.2 \pm 32.1$ & 0.091 \\
No. of transferred embryos & $1.8 \pm 0.4$ & $1.9 \pm 0.4$ & 0.123 \\
Clinical pregnancy rate & $70 / 122(57.4)$ & $78 / 150(52.0)$ & 0.376 \\
Implantation rate & $73 / 220(33.2)$ & $86 / 282(30.5)$ & 0.521 \\
Miscarriage rate & $11 / 73(15.1)$ & $14 / 86(16.3)$ & 0.897 \\
\hline
\end{tabular}

Values are presented as mean \pm standard deviation or number (\%). IVF, in vitro fertilization; ICSI, intracytoplasmic sperm injection. 
Table 3. Comparison of time-lapse parameters according to the fertilization method

\begin{tabular}{|c|c|c|c|c|c|}
\hline \multirow{2}{*}{$\begin{array}{l}\text { Development time } \\
\text { from t0 }(\mathrm{h})\end{array}$} & \multicolumn{2}{|c|}{ IVF $(n=809)$} & \multicolumn{2}{|c|}{ ICSI $(n=1,021)$} & \multirow{2}{*}{$p$-value } \\
\hline & Mean $\pm S D$ & 95\% Confidence interval & Mean $\pm S D$ & 95\% Confidence interval & \\
\hline tPNf & $25.2 \pm 4.2$ & $24.9-25.6$ & $24.3 \pm 3.9$ & $24.1-24.5$ & $<0.001$ \\
\hline $\mathrm{t} 2$ & $28.1 \pm 4.8$ & $27.7-28.5$ & $27.0 \pm 4.5$ & $26.8-27.3$ & $<0.001$ \\
\hline $\mathrm{t} 3$ & $37.1 \pm 6.1$ & $36.6-37.6$ & $36.5 \pm 5.7$ & $36.1-36.8$ & 0.021 \\
\hline t4 & $39.4 \pm 6.2$ & $38.9-39.9$ & $38.7 \pm 5.8$ & $38.3-39.0$ & 0.016 \\
\hline $\mathrm{t} 5$ & $49.9 \pm 8.8$ & $49.1-50.6$ & $48.7 \pm 7.9$ & $48.2-49.1$ & 0.005 \\
\hline t6 & $53.4 \pm 9.6$ & $52.6-54.2$ & $52.4 \pm 8.5$ & $51.9-52.9$ & 0.030 \\
\hline t7 & $56.0 \pm 10.1$ & $55.2-56.9$ & $55.6 \pm 9.6$ & $55.0-56.1$ & 0.358 \\
\hline t8 & $59.1 \pm 11.4$ & $58.1-60.0$ & $58.5 \pm 11.2$ & $57.9-59.2$ & 0.390 \\
\hline t9 & $71.1 \pm 13.2$ & $70.0-72.2$ & $70.7 \pm 13.2$ & $69.9-71.5$ & 0.669 \\
\hline $\mathrm{tM}$ & $91.1 \pm 11.5$ & $90.1-92.0$ & $91.0 \pm 11.8$ & $90.2-91.7$ & 0.924 \\
\hline $\mathrm{tSB}$ & $104.4 \pm 10.4$ & $103.5-105.3$ & $104.7 \pm 11.2$ & $104.0-105.4$ & 0.759 \\
\hline $\mathrm{tB}$ & $113.6 \pm 10.7$ & $112.7-114.5$ & $113.8 \pm 10.8$ & $113.2-114.5$ & 0.849 \\
\hline $\mathrm{tEB}$ & $120.1 \pm 10.9$ & $119.2-121.0$ & $121.7 \pm 12.0$ & $120.9-122.4$ & 0.344 \\
\hline $\operatorname{cc} 2(\mathrm{t} 3-\mathrm{t} 2)$ & $9.1 \pm 5.0$ & $8.7-9.4$ & $9.5 \pm 4.6$ & $9.2-9.8$ & 0.074 \\
\hline $\operatorname{cc} 3(\mathrm{t} 5-\mathrm{t} 3)$ & $12.9 \pm 6.3$ & $12.4-13.5$ & $11.9 \pm 6.9$ & $11.5-12.3$ & 0.001 \\
\hline $\mathrm{s} 1$ (t2-tPNf) & $2.6 \pm 0.8$ & $2.6-2.7$ & $2.6 \pm 1.0$ & $2.5-2.6$ & 0.069 \\
\hline $\mathrm{s} 2(\mathrm{t} 4-\mathrm{t} 3)$ & $2.5 \pm 4.9$ & $2.1-2.9$ & $2.2 \pm 3.9$ & $2.0-2.5$ & 0.251 \\
\hline s3 (t8-t5) & $10.0 \pm 9.8$ & $9.1-10.8$ & $10.9 \pm 12.9$ & $10.1-11.7$ & 0.124 \\
\hline
\end{tabular}

t0, insemination time; IVF, in vitro fertilization; ICSI, intracytoplasmic sperm injection; SD, standard deviation; tPNf, time to faded pronuclei; t2, time to 2 cells; $\mathrm{t} 3$, time to 3 cells; t4, time to 4 cells; t5, time to 5 cells; t6, time to 6 cells; t7, time to 7 cells; t8, time to 8 cells; $t 9$, time to 9 cells; tM, time to formation of a morula; $\mathrm{tSB}$, time to initiation of blastulation; $\mathrm{tB}$, time to the formation of the blastocyst; $\mathrm{tEB}$, time taken to complete the maximal blastocyst expansion.

Table 4. Abnormal early cleavage events

\begin{tabular}{lccc}
\hline Abnormal event & IVF $(n=809)$ & ICSI $(n=1,021)$ & $p$-value \\
\hline Direct cleavage from 1 to 3 cells & $189(23.4)$ & $210(20.6)$ & 0.149 \\
2 Multinucleated cells & $315(38.9)$ & $406(39.8)$ & 0.691 \\
2 Uneven cells & $171(21.1)$ & $201(19.7)$ & 0.463 \\
Reverse cleavage & $36(4.5)$ & $44(4.3)$ & 0.884 \\
\hline
\end{tabular}

Values are presented as number (\%).

IVF, in vitro fertilization; ICSI, intracytoplasmic sperm injection.

\section{Discussion}

This study is the largest retrospective study reported to date on the analysis of morphokinetic events and negative cleavage events associated with different insemination methods. In particular, this paper is the first to study the development of human embryos using a time-lapse system in Korea. The ICSI-based method selects one of the most suitable sperm cells based on morphology and motility under a microscope, and that sperm cell is injected into the cytoplasm of an oocyte to induce insemination. This method helps to overcome male infertility. However, a number of concerns have been raised about artificially removing the cumulus cells surrounding the oocyte and manipulating the oocyte while injecting the sperm cell into its cytoplasm. These hormonal and mechanical manipulations may have detrimental effects on subsequent embryo development and pregnancy [16]. In particular, compared to a cryo-IVF group, a higher major malformation rate was reported in a cryo-ICSI group [20], but this result is controversial.

Previous studies have reported that the initial development of ICSIderived embryos progressed faster than that of IVF-derived embryos. The 2-cell stage of the IVF embryos was 13 hours, compared to 10.5 hours for ICSI. Additionally, the 3-cell stage of ICSI embryos was 1.3 hours shorter than that of IVF embryos [7]. This suggests that IVF-derived embryos had higher synchrony during the second cleavage than ICSI-derived embryos. In one reported case, the 2-cell stage of IVF embryos (10.3 hours) was shorter than that of ICSI embryos (10.6 hours) [7]. However, according to a study that analyzed embryos that developed to late blastocysts within the 2-cell to 8-cell stages, ICSI not only had a faster $\mathrm{t} 2$, but also a faster $\mathrm{t} 3 \mathrm{than}$ standard IVF. AIthough ICSI showed faster development up to the 3-cell stage, there was no difference between the two groups from the 4-cell to 8-cell stages. Statistically significant differences were not found in the synchrony between $s 2$ and $s 3$ of the cell cycle [17]. This is similar to our results, which showed no significant differences between $\mathrm{s} 2$ and $\mathrm{s} 3$.

In our study comparing the developmental stages of IVF and ICSI, we found that the cleavage rate of ICSI was faster than that of IVF at $\mathrm{tPNF}, \mathrm{t} 2, \mathrm{t} 3, \mathrm{t} 4, \mathrm{t} 5$, and $\mathrm{t} 6$. Although no difference in $\mathrm{cc} 2$ was found, a significant difference in $\mathrm{CC} 3$ was found between IVF and ICSI (12.9 hours vs. 11.9 hours, $p<0.05$ ).

In a retrospective study of 1,203 cleavage-stage embryos inseminated based on the oocyte donation model [21], the cleavage rate of 
ICSI was found to be slightly faster than that of IVF at tPNF, t2, t5, t7, and t9. A sperm cell must pass through cumulus cells and the zona pellucida to fuse with the oolemma for insemination to occur in conventional IVF, whereas ICSI bypasses such processes. These differences in the process explain why IVF embryos have a slower initial development time than ICSI embryos.

The major developmental events and implantation rates were studied using time-lapse imaging [22], and it was found that the 2to 3-cell stages lasted for less than 5 hours in approximately $14 \%$ of embryos. The implantation rate was low, approximately $1 \%$ to $2 \%$, when these embryos were used for transplantation. For this reason, direct cleavage from 1 to 3 cells could be considered as an exclusion criterion [10]. In a retrospective study of 834 embryos using different insemination methods, differences in direct cleavage were tested [21]; ICSI-derived embryos had a higher direct-cleavage rate than IVF-derived embryos (11.1\% vs. $10.0 \%$, respectively), but the result was not statistically significant. Interestingly our study was different in that IVF-derived embryos showed a slightly higher direct-cleavage rate than ICSI-derived embryos (23.4\% vs. $20.6 \%$, respectively), but the difference was likewise not statistically significant.

IVF-ET has made a leap forward. The introduction of time-lapse techniques has made it possible to observe embryonic development, detect abnormal cleavage events, and respond with clinical treatments. Multinucleated blastomeres result from abnormal cleavage and may be detrimental to late embryonic development or clinical outcomes, and they are a key criterion that cannot be excluded from the embryo grading system [23]. Previous studies have reported that multinucleated blastomeres increased the aneuploidy rate and decreased the implantation rate [24-26]. Another retrospective study of 2-cell multinucleated embryos and non-multinucleated embryos was performed on 550 patients, and the results showed that multinucleated embryos had a lower clinical pregnancy rate $(23.4 \%$ vs. $44.0 \%$ ) and a lower implantation rate ( $23.3 \%$ vs. $43.6 \%)$ than nonmultinucleated embryos. The multinucleated blastomeres had worse clinical outcomes [27]. However, in our study, no significant difference was found between the IVF and ICSI groups with regard to 2-cell multinucleation events or clinical outcomes.

Uneven blastomere cleavage and other major abnormalities are known to have negative effects on clinical outcomes. According to a retrospective study performed on 378 transplanted embryos [28], uneven blastomere cleavage was associated with a lower pregnancy rate (37.6\% vs. $52.9 \%$, respectively) and a lower implantation rate (23.9\% vs. $36.4 \%$, respectively) than even blastomere cleavage. In our comparative study of ICSI and IVF, there was no significant difference in the incidence of uneven blastomere cleavage between the two groups, and there was no difference in the clinical outcomes. This is the first study to report the incidence of even blastomere cleavage using a time-lapse system.

In conclusion, ICSI bypasses a specific process that is included in conventional IVF, which results in a differentiation process that is 1 hour faster on average until the 6-cell stage. However, no significant differences were observed from that point until the blastocyst stage. Additionally, no significant differences in abnormal cleavage events, such as direct cleavage, the multinucleation of 2 cells, and uneven blastomere cleavage, were found between the ICSI and IVF groups, which explains why no significant differences were found in the clinical outcomes between the two groups.

\section{Conflict of interest}

No potential conflict of interest relevant to this article was reported.

\section{References}

1. Pinborg A, Loft A, Schmidt L, Andersen AN. Morbidity in a Danish national cohort of 472 IVF/ICSI twins, 1132 non-IVF/ICSI twins and 634 IVF/ICSI singletons: health-related and social implications for the children and their families. Hum Reprod 2003;18: 1234-43.

2. Seoud MA, Toner JP, Kruithoff C, Muasher SJ. Outcome of twin, triplet, and quadruplet in vitro fertilization pregnancies: the Norfolk experience. Fertil Steril 1992;57:825-34.

3. Lieberman B. An embryo too many? Hum Reprod 1998;13:26646.

4. Cutting R, Morroll D, Roberts SA, Pickering S, Rutherford A; BFS and ACE. Elective single embryo transfer: guidelines for practice British Fertility Society and Association of Clinical Embryologists. Hum Fertil (Camb) 2008;11:131-46.

5. Kirkegaard K, Hindkjaer JJ, Grondahl ML, Kesmodel US, Ingerslev $\mathrm{HJ}$. A randomized clinical trial comparing embryo culture in a conventional incubator with a time-lapse incubator. J Assist Reprod Genet 2012;29:565-72.

6. Montag M, Liebenthron J, Koster M. Which morphological scoring system is relevant in human embryo development? Placenta 2011;32 Suppl 3:S252-6.

7. Lemmen JG, Agerholm I, Ziebe S. Kinetic markers of human embryo quality using time-lapse recordings of IVF/ICSI-fertilized oocytes. Reprod Biomed Online 2008;17:385-91.

8. Nakahara T, Iwase A, Goto M, Harata T, Suzuki M, lenaga M, et al. Evaluation of the safety of time-lapse observations for human embryos. J Assist Reprod Genet 2010;27:93-6.

9. Cruz M, Gadea B, Garrido N, Pedersen KS, Martinez M, Perez-Cano I, et al. Embryo quality, blastocyst and ongoing pregnancy rates in oocyte donation patients whose embryos were moni- 
tored by time-lapse imaging. J Assist Reprod Genet 2011;28:56973.

10. Meseguer M, Herrero J, Tejera A, Hilligsoe KM, Ramsing NB, Remohi J. The use of morphokinetics as a predictor of embryo implantation. Hum Reprod 2011;26:2658-71.

11. Payne D, Flaherty SP, Barry MF, Matthews CD. Preliminary observations on polar body extrusion and pronuclear formation in human oocytes using time-lapse video cinematography. Hum Reprod 1997;12:532-41.

12. Mio Y. Morphological analysis of human embryonic development using time-lapse cinematography. J Mamm Ova Res 2006; 23:27-35.

13. Mio Y, Maeda K. Time-lapse cinematography of dynamic changes occurring during in vitro development of human embryos. Am J Obstet Gynecol 2008;199:660.e1-5.

14. Nagy ZP, Liu J, Joris H, Devroey P, Van Steirteghem A. Timecourse of oocyte activation, pronucleus formation and cleavage in human oocytes fertilized by intracytoplasmic sperm injection. Hum Reprod 1994;9:1743-8.

15. Plachot M. Fertilization. Hum Reprod 2000;15 Suppl 4:19-30.

16. Bar-Hava I, Ashkenazi J, Shelef M, Schwartz A, Brengauz M, Feldberg $D$, et al. Morphology and clinical outcomes of embryos after in vitro fertilization are superior to those after intracytoplasmic sperm injection. Fertil Steril 1997;68:653-7.

17. Dal Canto M, Coticchio G, Mignini Renzini M, De Ponti E, Novara PV, Brambillasca F, et al. Cleavage kinetics analysis of human embryos predicts development to blastocyst and implantation. Reprod Biomed Online 2012;25:474-80.

18. Kim HJ, Yoon HJ, Jang JM, Oh HS, Lee YJ, Lee WD, et al. Comparison between intracytoplasmic sperm injection and intracytoplasmic morphologically selected sperm injection in oligo-astheno-teratozoospermia patients. Clin Exp Reprod Med 2014; 41:9-14.

19. Son WY, Yoon SH, Yoon HJ, Lee SM, Lim JH. Pregnancy outcome following transfer of human blastocysts vitrified on electron microscopy grids after induced collapse of the blastocoele. Hum
Reprod 2003;18:137-9.

20. Belva F, Henriet S, Van den Abbeel E, Camus M, Devroey P, Van der Elst J, et al. Neonatal outcome of 937 children born after transfer of cryopreserved embryos obtained by ICSI and IVF and comparison with outcome data of fresh ICSI and IVF cycles. Hum Reprod 2008;23:2227-38.

21. Cruz M, Garrido N, Gadea B, Munoz M, Perez-Cano I, Meseguer M. Oocyte insemination techniques are related to alterations of embryo developmental timing in an oocyte donation model. Reprod Biomed Online 2013;27:367-75.

22. Rubio I, Kuhlmann R, Agerholm I, Kirk J, Herrero J, Escriba MJ, et al. Limited implantation success of direct-cleaved human zygotes: a time-lapse study. Fertil Steril 2012;98:1458-63.

23. Yakin K, Balaban B, Urman B. Impact of the presence of one or more multinucleated blastomeres on the developmental potential of the embryo to the blastocyst stage. Fertil Steril 2005;83: 243-5.

24. Ambroggio J, Gindoff PR, Dayal MB, Khaldi R, Peak D, Frankfurter $D$, et al. Multinucleation of a sibling blastomere on day 2 suggests unsuitability for embryo transfer in IVF-preimplantation genetic screening cycles. Fertil Steril 2011;96:856-9.

25. Kligman I, Benadiva C, Alikani M, Munne S. The presence of multinucleated blastomeres in human embryos is correlated with chromosomal abnormalities. Hum Reprod 1996;11:1492-8.

26. Van Royen E, Mangelschots K, Vercruyssen M, De Neubourg D, Valkenburg M, Ryckaert G, et al. Multinucleation in cleavage stage embryos. Hum Reprod 2003;18:1062-9.

27. Ergin EG, Caliskan E, Yalcinkaya E, Oztel Z, Cokelez K, Ozay A, et al. Frequency of embryo multinucleation detected by timelapse system and its impact on pregnancy outcome. Fertil Steril 2014;102:1029-33.e1.

28. Hardarson T, Hanson C, Sjogren A, Lundin K. Human embryos with unevenly sized blastomeres have lower pregnancy and implantation rates: indications for aneuploidy and multinucleation. Hum Reprod 2001;16:313-8. 